\title{
Channel Dependent Interference and Decentralized Colouring
}

\author{
P. Clifford, D. J. Leith \\ Hamilton Institute, NUI Maynooth, Ireland
}

\begin{abstract}
We consider channel allocation to mitigate interference between wireless LANs. The channel allocation task is often formulated in the literature as finding a proper colouring of a single graph. We show that the interference between WLANs can be channel dependent in which case a different conflict graph is associated with each channel. Channel allocation then corresponds to a multi-graph colouring problem. This potentially has profound implications as the behaviour of many proposed colouring-based algorithms for channel allocation is unclear in a multi-graph context. We prove that a recently proposed decentralized colouring algorithm performs correctly in the multi-graph setting. We also present a new, extended version of this algorithm suited to a wide range of multi-radio architectures.
\end{abstract}

\section{Introduction}

We consider how a group of access-points/base-stations ${ }^{1}$ can configure their channel choice so as to minimise interference between one another. This problem has recently been the subject of an upsurge of interest in the WLAN literature, e.g. see [2-13]. The channel allocation task is often formulated in the literature as finding a proper colouring of a single graph. That is, a conflict graph is constructed by associating a graph vertex with each WLAN and inserting edges between WLANs that interfere. A non-interfering channel allocation then corresponds to a proper colouring of this conflict graph. We demonstrate that this formulation may be unrealistic. Specifically, we show that the interference between WLANs can be channel dependent in which case a different conflict graph is associated with each channel. Channel allocation then corresponds to a multi-graph colouring problem. This potentially has profound implications as the behaviour of many proposed colouring-based algorithms for channel allocation is unclear in a multi-graph context.

Our second main contribution is to establish that a recently proposed decentralized colouring algorithm does indeed generalise to the multi-graph setting. We also present a new, extended version of this algorithm suited to a wide range of multi-radio architectures.

\footnotetext{
${ }^{1}$ We use the term access point or AP to denote the co-ordinating station in a WLAN that is responsible for channel selection. Consideration is not restricted to a specific WLAN technology. Each AP has associated wireless client stations and we refer to the collection of clients plus AP as a WLAN.
} 


\section{Channel allocation and graph colouring}

The channel allocation task is usually formulated as a standard graph colouring problem. For example, Figure 1 shows four interfering WLANs. Transmissions within the AP1 and AP2 WLANs can interfere, with the interference range of each WLAN indicated by the dashed circles in Figure 1. The level of interference between any particular pair of transmissions depends on the physical locations of the communicating stations. This can easily lead to complex hidden/exposed terminal problems. For example, if AP2 transmits data to client 1 at the righthand edge of the figure at the same time as the client 2 station located at the left-hand edge of the figure sends data to AP1, then reception by AP1 may be blocked by AP2's transmission while AP2's transmission is successfully received at the right-hand station as this is beyond the interference range of AP1. This is, of course, an example of hidden terminal behaviour, known to have the potential to induce gross unfairness and reduced network utilisation. Similarly AP3 and AP4's transmissions can interfere creating further potential for four-way hidden/exposed terminal behaviour.

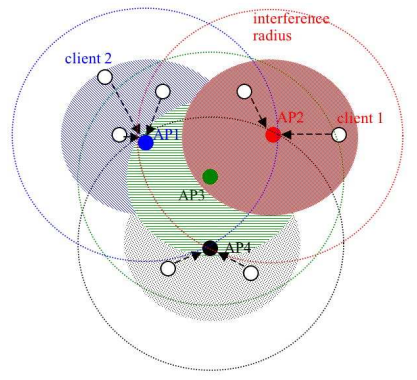

Fig. 1. Example of interfering 802.11 WLANs. Dashed circles indicate interference radius, shaded circles indicate communication radius.

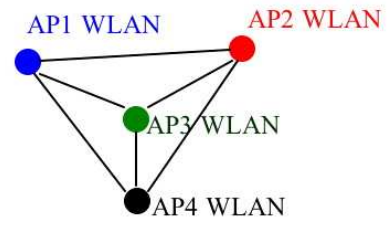

Fig. 2. Interference graph of Figure 1.

The underlying channel selection problem in this example is equivalent to graph colouring. To see this, define the interference graph by associating a node with each WLAN (e.g. with each BSS in an 802.11 network) and inserting an edge between nodes that interfere. For example, Figure 2 shows the interference graph corresponding to the wireless network in Figure 1. A colouring of the graph assigns colours to each node, and a proper colouring is an assignment of colours to each node such that no adjacent nodes share the same colour. A non-interfering channel allocation is then equivalent to a proper colouring of the interference graph associated with a wireless network. Similar considerations also apply in multi-hop multi-radio situations. For example, AP3 might be a multiradio intermediate relay station with $\mathrm{AP} 4$ the only access point with a wired backhaul link. 


\section{Channel dependent interference}

It is important to stress here that the use of circles to denote interference regions in Figure 1 is an idealisation. Importantly, we note that since channel characteristics are dependent on the frequency used, we can expect that the shape of the interference regions will be channel dependent.

To investigate this question, we took measurements on an experimental testbed. The testbed consists of 10 PC-based embedded Linux boxes based on the Soekris net4801, 5 boxes configured as APs in infrastructure mode and 5 as client stations. We also use 5 PCs acting as monitoring stations to collect measurements - this is to ensure that there is ample disk space, RAM and CPU resources available so that collection of statistics does not impact on the transmission of packets. These machines are setup as five WLANs (denoted WLAN A - WLAN E) located in a university office space. All systems are equipped with an Atheros $802.11 \mathrm{a} / \mathrm{b} / \mathrm{g}$ mini-PCI card with an external antenna. All nodes use a Linux 2.6.16.20 kernel and the MADWiFi wireless driver. All of the systems are also equipped with a 100Mbps wired Ethernet port, which is used for control of the testbed from a PC. Specific vendor features on the wireless card, such as turbo mode and channel scanning, are disabled.

The testbed hardware supports operation both in the $802.11 \mathrm{a} 5 \mathrm{GHz}$ band and in the $802.11 \mathrm{~b} 2.4 \mathrm{GHz}$ band. While spectrum analyzer measurements revealed little external interference in the $5 \mathrm{GHz}$ band (a noise floor of around $-80 \mathrm{~dB}$ being typical), significant external interference was observed in the $2.4 \mathrm{GHz}$ band which is attributed to bluetooth devices . Focussing on the $5 \mathrm{GHz}$ band, our measurements indicated that the level of interference between WLANs can be strongly channel dependent. For example, the measured interference level between WLANs B and C as the channel number is varied (with WLANs B and C always sharing the same channel), varied from 0 on channel 36 , to $27 \%$ on channel 56 , and back to $1 \%$ on channel 64 . This behaviour is perhaps unsurprising as we can expect path propagation characteristics to be frequency dependent. Nevertheless, it has profound implications for channel allocation algorithms. In particular, it is in general not sufficient to confine consideration to a single conflict graph as shown for example in Figure 2, but rather a different conflict graph may be associated with each available frequency channel. An immediate consequence is that the channel allocation problem is not necessarily equivalent to the standard colouring task on a single graph, but rather may involve a more general multi-graph colouring task.

\section{Implications for channel allocation algorithms}

The chromatic number (minimum number of colours for a proper colouring) of the multi-graph problem is only weakly related to the constituent individual graphs. We illustrate this by example.

Figure 3 shows the conflict graphs associated with channels 1,2 and 3 in a network of 6 interfering WLANs and also shows a successful channel allocation 


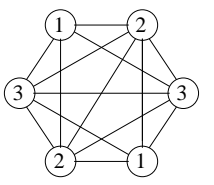

Channel 1

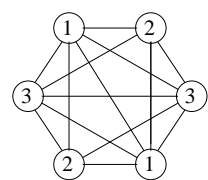

Channel 2

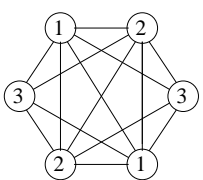

Channel 3

Fig. 3. Multi-graph example 1. Individual channel conflict graphs shown with proper colouring requiring only 3 colours.

using 3 channels. Although for each channel there is only one pair of nodes which do not interfere, the arrangement is such that only three channels are necessary to avoid interference, rather than the six which would be required if every node interfered with every other on every channel. This example demonstrates that the problem of multi-graph colouring is dramatically different to normal graph colouring. To our knowledge, no analytic results are available on the performance of colouring algorithms on multi-graphs. Existing convergence proofs for distributed algorithms such as those in $[8,10,13]$ relate to colouring of a single graph. Centralised channel allocation algorithms based on single graph colouring may exhibit unexpected behaviour in a multi-graph context.

Channel dependent interference also has direct implications for frequency hopping approaches to channel allocation such as that in [5] and elsewhere. The performance of heuristic algorithms is unclear.

\section{$5 \quad$ Main Result}

We refer to [13] for the decentralized channel allocation algorithm (Section 6 in this paper contains a generalisation). Let $G(i)=(V, E(i))$ denote the interference graph associated with use of channel $i$ in a wireless network. That is, the vertices $V$ of $G(i)$ are the network WLANs and the edge set $E(i)$ contains an edge between vertices $(u, v)$ when WLAN $u$ and $v$ interfere on channel $i$. The interference environment is then characterised by the family of graphs $\{G(i), i \in[1,2, . ., c]\}$. A non-interfering channel allocation is one where each WLAN uses a channel $i$ that is different from all of its neighbours in $G(i)$. Note that in the special case where $G(i)=G \forall i$ then the interference graph is the same on every channel and we recover a standard single graph colouring problem.

Theorem 1. Suppose each vertex in $V$ operates the CFL algorithm. Assume that the channel allocation problem is feasible (i.e. a non-interfering channel allocation does indeed exist). Then the CFL algorithm converges, with probability one, to a non-interfering channel allocation.

Our proof also provides a partial answer to a further question, namely how quickly the algorithm converges to a non-interfering allocation. The stopping time is the time taken for the algorithm to converge. We have the following property. 
Corollary 1. Let $\tau$ denote the stopping time of the CFL algorithm. Then prob $[\tau>$ $k]<\alpha e^{-\gamma k}$, for positive constants $\alpha, \gamma$.

Our argument does not yield a tight estimate of the exponent $\gamma$, which determines the precise convergence rate of the algorithm, but given that the underlying colouring problem is NP-hard this is unsurprising. Extensive simulations not presented here demonstrate that the convergence is rapid on average, similarly to the simulations presented in [13] for the single graph case.

We will show that in a determined finite amount of steps the system has some minimum positive probability of convergence. We show that starting from any configuration the system can reach some standard state after two steps. From this standard state we show that the system can then potentially reach a state where every node experiences a failure simultaneously, allowing convergence without issues of dependence between nodes. Hence the network always has positive probability of global success and so will almost surely converge.

In the sequel we refer to two nodes choosing the same channel as a "collision". We say that the state $\mathbb{S}$ consists of the set of all possible configurations where (i) the channel selections of at least two nodes interfere and (ii) at all colliding nodes the selection probability for every channel is bounded away from zero (in fact, we will consider the case where they are strictly greater than $\left.\frac{b(1-b)}{c-1}\right)$. We define the master graph: an edge is in the master graph if it is in any of the individual channel graphs $G(i), i \in[1,2, . ., c]$. Denote the maximum node degree of the master graph by $m d$ and the diameter of the master graph (length of the longest shortest path between two nodes) by $D$.

Consider a colliding node. Observe from [14] that a node colliding on one colour and then on a different colour ensures that its selection probabilities for all channels are strictly greater than $b(1-b) /(c-1)$. Similarly if a node succeeds and then collides. However, it can be seen that repeated collisions on the same channel can result in the channel selection probability becoming arbitrarily small. Thus, the system may avoid state $\mathbb{S}$ by some node undergoing repeated same channel collisions. We show in Lemma 1 that if the system has reached a configuration with some channel selection probabilities lower than $b(1-b) /(c-1)$ at one or more colliding nodes, then there is a positive lower-bounded probability that it will return in two steps to our standard state $\mathbb{S}$. The following Lemma is proved in [14].

Lemma 1. From any configuration of the system, if after two steps the system has not converged, it is in state $\mathbb{S}$ with some probability $p r_{5}>0$.

We proceed by defining the directed graph $D G$ which is dependent on the current channel selection by the network nodes. There is an edge in $D G$ from node $u$ to node $v$ if an edge exists between $u$ and $v$ in the graph $G\left(i_{v}\right)$ where $i_{v}$ is the channel currently chosen by node $v$. We say $v$ is a $D G$-neighbour of $u$. The edges directed into a node $v$ are determined by the channel selection of that node together with the conflict graphs $G$, but are unaffected by the channel selections of other nodes. Existence of a directed path in graph $D G$ from node $u$ to node $v$ indicates that the node $u$ can potentially force a collision at node $v$ 
(by first generating a collision with its immediate neighbour, which in turn can generate a collision with its neighbour, and so on until node $v$ is reached).

$D G$-graphs associated with an example network are illustrated in Figure 5. Consider the lower left node. Edges involving this node only exist on channel R. Hence, this node can potentially create collisions with its neighbours by selecting channel R. However, by selecting channel B, the lower left node can always avoid interference from any of the other nodes regardless of their channel selection. This asymmetric nature of the relationship between the lower left node and its neighbours is indicated by the directional arrows on the $D G$-graph links.

Note also that once it chooses channel B, the lower left node in Figure 5 is unreachable from the other nodes. Since it is unreachable, no collisions can occur, choice of channel B will yield a "success" in the CFL algorithm and the node will remain on channel B thereafter i.e. the node will be converged and permanently unreachable. That is, the CFL algorithm therefore ensures that unreachable nodes remain permanently unreachable. A second example illustrating this behaviour is also given in the right-hand graphs in Figure 5. These examples illustrate the general point that as the CFL algorithm proceeds connectivity can change and, in particular, certain nodes may become permanently unreachable and we need to take account of this when analyzing convergence.

We define the set of nodes $C N$ to be all nodes which are unreachable from any node which just collided. We note that any node $w \in C N$ must have just been successful. In addition, no matter what colour choices other nodes make in the future, $w$ will never subsequently undergo a collision (since $w$ is unreachable). Hence any nodes in $C N$ are converged and can be ignored for the remainder of the proof. Note that the graph $D G$ changes as the algorithm proceeds, and nodes can join $C N$ but will never leave. In Figure 5 we see two stages of the algorithm, the corresponding $D G$, and the set $C N$ illustrated by nodes in bold.

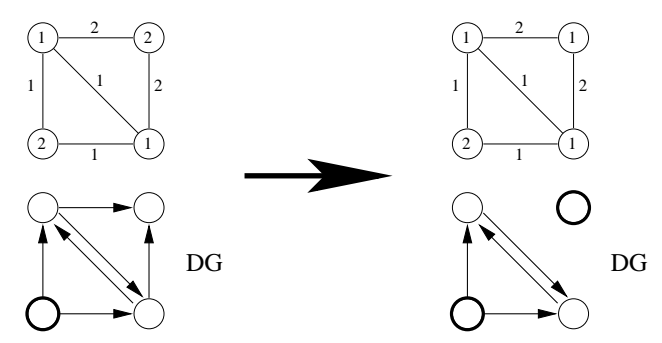

Fig. 4. Illustrating definition of DG-graphs. Upper graphs show node channel selections and the channel-dependence of edges is indicated by labels. Lower graphs show corresponding DG-graphs. The set $C N$ indicated by nodes in bold.

Lemma 2. Suppose that the system is in state $\mathbb{S}$. There exists a specific evolution $\mathbb{E}$ of the system which results in all nodes not in $C N$ colliding. 
Proof of Lemma 2. Consider one of the collisions. Two nodes $k_{1}$ and $k_{2}$, say, have just experienced a collision. By way of notational convenience we say these two nodes were visited at step 2. Suppose now that $k_{1}$ collides with its first non visited $D G$-neighbour $k_{3}$ (if any) at step 3 . Suppose also that $k_{2}$ collides with its first non visited $D G$-neighbour (if any, potentially $k_{3}$ also) at step 3 also. We say that such nodes are visited at step 3. Inductively suppose now that a node once visited collides with all its nonvisited $D G$-neighbours in consecutive steps. This is possible because a visited node having just collided can potentially choose any channel. Note that a node being visited simultaneously (along two different equal length paths from $k_{1}$ and $k_{2}$ say) is also possible.

Suppose that once a node has collided with all its nonvisited neighbours it then repeatedly chooses channel 1 until step $T_{1}=T_{0}+3+m d \times D$. We note that as a node $k_{4}$ is colliding with its nonvisited $D G$-neighbours some of them may become visited from other nodes before they collide with $k_{4}$; we suppose then that $k_{4}$ does not visit such nodes.

Concurrently with this visiting procedure starting at the nodes $k_{1}$ and $k_{2}$, we can suppose that the same visiting procedure starts at all nodes in $J C$, and traverses the graph as before. Again as a node $k_{5}$ is colliding with its nonvisited $D G$-neighbours some of them may become visited from other nodes, and we again suppose that $k_{5}$ does not visit such nodes.

When all the visited nodes have visited all their neighbours, every node not in $C N$ has been visited and is choosing channel 1. Some nodes which are now choosing channel 1 may of course have entered the set $C N$ and are ignored. Hence every node not in $C N$ is colliding. At the next time step we suppose that every node chooses a colour so that no collisions occur.

Lemma 3. Suppose that there exists a choice of channels that yields a noninterfering allocation. There is a strictly positive lower bound $p r_{8}$ on the probability of the evolution $\mathbb{E}$ occurring from any configuration in state $\mathbb{S}$.

Proof of Lemma 3. Given the initial colour selection probabilities and the set $J C$, the evolution $\mathbb{E}$ is well defined. The duration of $\mathbb{E}$ is at most $m d \times D$ timesteps. Hence $\mathbb{E}$ has some positive (computable) probability $p r_{6}$ of occurring since the system is finite.

By assumption the system begins in state $\mathbb{S}$ and so the initial colour selection probabilites of just collided nodes are lower bounded; therefore there is some probability $p r_{7}>0$ such that $p r_{6}>p r_{7}$ irrespective of the initial colour selection probabilities.

The set $J C$ is one of finitely many possibilities and so again there is some probability $p r_{8}>0$ such that $p r_{7}>p r_{8}$ irrespective of the initial choice of $J C$.

Proof of Theorem 1. Defining $p r_{9}=p r_{8} p r_{5}$ gives the probability that the system is in state $\mathbb{S}$ after the first two steps and then follows evolution $\mathbb{E}$. Hence every $2+m d \times D$ steps the system will converge with probability at least $p r_{9}$. Hence after $j(2+m d \times D)$ steps we have converged with probability at least $1-\left(1-p r_{9}\right)^{j}$ which converges to 1 as $j \rightarrow \infty$. 


\section{Multiple radios}

The use of wireless access points equipped with multiple radios has been the subject of much recent interest. The CFL algorithm can be applied without change to multi-radio access points by running a separate copy of the CFL algorithm for each radio. This will yield a non-interfering channel allocation for every radio. In this section we illustrate that the CFL algorithm can be further generalised to take explicit account of bit rate requirements in a multi-radio setting.

Specifically, we consider the following task. Suppose we have a set of interfering WLANs (possibly with channel-dependent interference) and a set $\mathcal{C}$ of available channels. Let $b_{i}$ denote the bit rate associated with channel $i$. At access point $j$ we require to select a non-interfering set of channels $C \subseteq \mathcal{C}$ such that $\sum_{i \in C} b_{i} \geq B$ and with cardinality $|C| \leq r$, where $r$ is the number of radios at the access point. Note that the channel bit rate $b_{i}$, the target bit rate $B$, number of radios $r$ and set of available channels $\mathcal{C}$ may be different for each access point.

The change here over our previous discussion is the inclusion of the bit rate constraint $\sum_{i \in C} b_{i} \geq B$. Such a bit rate requirement arises, for example, when striping data across multiple radios. One advantage over simply allocating a channel to every available radio is that it may be that fewer radios are sufficient to provide the required bandwidth, thereby reducing the load on the spectrum in dense WLAN deployments. This formulation also allows us to take explicit account of the different quality of each channel - this can be important in multiradio settings where radios are heterogeneous e.g. some radios might be 802.11 based and others 802.16 based. We note that the bit-rate constrained channel allocation problem is also relevant to dynamic spectrum management in wired DSL lines (where cross-talk across wiring bundles is a significant source of interference) [15]. We introduce the following generalised version of the CFL algorithm to solve the multiple radio bit-rate constrained channel allocation problem.

Let $c$ denote the number of available channels at an access point and the access point maintain a $c$ element state vector $p$ with element $p_{i}$ corresponding to the probability of transmitting on the $i$ th channel. Since we allow use of multiple radios, note that we do not require the $p_{i}$ 's to sum to one. Consider the following decentralized algorithm for updating $p$.

\section{Generalised CFL Algorithm}

1. Initialise $p=[r / c, r / c, \ldots, r / c]$

2. Pick a random ordering of the channels. In that order, toss coins to activate channel $i$ with probability $p_{i}$. Stop immediately once the AP's target bit rate is met. This results in a set $C$ of active channels.

3. If $\sum_{i \in C} b_{i}<B$, multiply every probability by $1+b$, set $C=\emptyset$, and repeat ${ }^{2}$ step 2. We note that the random selection process at step 2 above together with the redistribution of probability in step 6 below, ensures that there is

\footnotetext{
${ }^{2}$ The precise procedure here is not important. The feasible active set may be found in any reasonable fashion provided any channel with nonzero $p_{i}$ might be active and that channels with larger $p_{i}$ are more likely.
} 

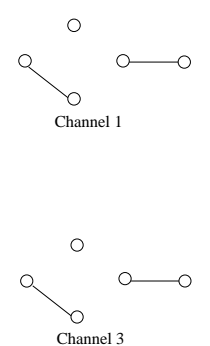

Fig. 5. Five AP example of multiple radios. Each graph gives the interference for a particular channel.
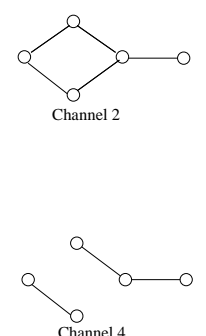

$1,3,4 \bigcirc$

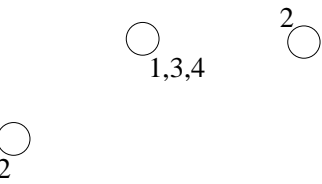

2

2

Fig. 6. Possible final result of multiple radio algorithm. The figure shows a successful channel allocation.

a positive lower bound on the probability of any feasible allocation after a collision.

4. Sense the quality of the channels in set $C$. We obtain "success" on channel $i \in C$ if this does not interfere with any neighbouring WLAN and otherwise have a "failure".

5. If we have success on all active channels, update $p$ as

$$
p_{i}=1 \forall i \in C, p_{j}=0 \forall j \notin C
$$

i.e. on a successful choice we use the same set of channels for the next round. This ensures that any channel allocation that satisfies the target bit rate and also removes interference between all WLANs is an absorbing state.

6 . Otherwise let $S$ denote the set of channels which were successful, $F$ the set of failed channels and $I$ the set of inactive channels. Update $p$ as

$$
\begin{aligned}
& p_{i}=1 \forall i \in S, \\
& p_{j}=(1-b) p_{j}+b \forall j \in I, \\
& p_{k}=(1-b) p_{k} \forall k \in F .
\end{aligned}
$$

The lower bound on these probabilities after the node fails is much more important than the exact choice of parameters.

\section{Return to 2}

This algorithm maintains the three key properties of the original CFL algorithm, namely (i) that if every WLAN is successful the system remains in this successful configuration henceforth; (ii) after a collision any feasible channel allocation is possible; and (iii) if one WLAN is failing the failure can propagate to neighbouring WLANs and force them (with some probability) to change their channel allocation. Hence by a similar proof to that for Theorem 1 the generalized CFL algorithm will converge with probability 1 to a non-interfering channel allocation satisfying the specified bit rate requirements, provided one exists.

In Figure 5 we present an example of the multiple radio problem. Suppose that every AP has bit rate demand 3 units; suppose that channel 2 has bit rate 3 units and that all other channels have bit rate 1 unit. Figure 6 illustrates a feasible channel allocation which is a result of the algorithm. 


\section{Conclusions}

We show that the interference between WLANs can be channel dependent in which case a different conflict graph is associated with each channel. This potentially has profound implications as the behaviour of proposed colouring-based algorithms for channel allocation is unclear in a multi-graph context. We are, however, able to show that a recently proposed decentralized colouring algorithm does generalise to the multi-graph setting. We also present a new, extended version of this algorithm suited to a wide range of multi-radio architectures. This work was supported by Science Foundation Ireland grant IN3/03/I346.

\section{References}

1. L. Tassiulas, A. Ephremides, "Stability properties of constrained queueing systems and scheduling policies for maximum throughput in multihop radio networks", IEEE Trans Automatic Control, 37 (12), 1992.

2. A. Akella, G. Judd, P. Steenkiste, and S. Seshan. "Self management in chaotic wireless deployments". In MobiCom, 2005

3. H. Luo, P. Medvedev, J. Cheng, S. Lu, "A self coordinating approach to distributed fair queuing in ad hoc wireless networks", Proc. of IEEE INFOCOM '01, 2001.

4. A. Raniwala, T. Chiueh. "Architecture and algorithms for an IEEE 802.11-based multi-channel wireless mesh network". In Proc IEEE International Conference on Computer Communications, 2005.

5. A.Mishra, V.Shrivastava, D.Agarwal, S.Banerjee, S.Ganguly, Distributed Channel Management in Uncoordinated Wireless Environments. In MobiCom, 2006.

6. A.Mishra, V.Brik, S.Banerjee, A.Srinivasan, W.Arbaugh, "A Client-Driven Approach for Channel Management". Proc. IEEE INFOCOM, 2006

7. B.J. Leung, K.K. Kim, "Frequency assignment for IEEE 802.11 wireless networks". Proc. 58th IEEE Vehicular Technology Conference, 2003.

8. B. Kauffmann, F. Baccelli, A. Chaintreau, K. Papagiannaki, C. Diot, "Self Organization of Interfering 802.11 Wireless Access Networks,", INRIA Technical Report, August 2005.

9. A. Subramanian, H. Gupta and S. R. Das, "Minimum interference channel assignment in multi-radio wireless mesh networks" Proc. Mobicom, 2006.

10. B.J.Ko, V.Mishra, J.Padhye, D.Rubenstein, "Distributed channel assignment in multi-radio 802.11 mesh networks". http://www1.cs.columbia.edu/ danr/publish/2006/jun-tr06.pdf

11. A. K. Das, S. Roy and R. Vijaykumar, "Static Channel Assignment in Multiradio Multi-channel 802.11 Wireless Mesh Networks: Issues, Metrics and Algorithms". Proc. IEEE Globecom, 2006

12. K. Ramachandran, E. Belding, K. Almeroth, M. Buddhikot, "Interference-Aware Channel Assignment in Multi-Radio Wireless Mesh Networks". Proc. IEEE INFOCOM, 2006.

13. D.J. Leith, P. Clifford, "A Self-Managed Distributed Channel Selection Algorithm for WLANs". Proc. ACM/IEEE RAWNET, Boston, 2006.

14. D.J. Leith, P. Clifford, "Convergence of Distributed Learning Algorithms for Optimal Wireless Channel Allocation". Proc. IEEE CDC, San Diego, 2006.

15. T. Starr, J.M. Cioffi, P. Silverman, "Understanding Digital Subscriber Lines", Prentice Hall: Upper Saddle River, NJ, 1999. 